\title{
Short term outcomes of prostate biopsy in men tested for cancer by prostate specific antigen: prospective evaluation within ProtecT study
}

\author{
(c) $(1)$ (8) OPEN ACCESS
}

\begin{abstract}
Derek J Rosario senior lecturer in cancer studies ${ }^{1}$, J Athene Lane senior research fellow ${ }^{2}$, Chris Metcalfe senior lecturer in medical statistics ${ }^{2}$, Jenny L Donovan professor of social medicine ${ }^{2}$, Andy Doble consultant urological surgeon ${ }^{3}$, Louise Goodwin research nurse and biobanker ${ }^{1}$, Michael Davis research associate in health services research ${ }^{2}$, James W F Catto reader in cancer studies ${ }^{1}$, Kerry Avery research fellow and NIHR post-doctoral award holder ${ }^{2}$, David E Neal professor of surgical oncology ${ }^{4}$, Freddie C Hamdy professor of surgery ${ }^{5}$
\end{abstract}

\footnotetext{
${ }^{1}$ Academic Urology Unit, Department of Oncology, Royal Hallamshire Hospital, University of Sheffield, Sheffield S10 2JF, UK; ${ }^{2}$ School of Social and Community Medicine, University of Bristol, Bristol BS8 2PS, UK; ${ }^{3}$ Department of Urology, Addenbrooke's Hospital, Cambridge CB2 0QQ, UK; ${ }^{4}$ Department of Oncology, University of Cambridge, Addenbrooke's Hospital; ${ }^{5}$ Nuffield Department of Surgical Sciences, University of Oxford, John
} Radcliffe Hospital, Oxford OX3 9DU, UK

\begin{abstract}
Objectives To measure the effect of the adverse events within 35 days of transrectal ultrasound guided biopsy from the perspective of asymptomatic men having prostate specific antigen (PSA) testing; to assess early attitude to re-biopsy; to estimate healthcare resource use associated with adverse events due to biopsy; and to develop a classification scheme for reporting adverse events after prostate biopsy. Design Prospective cohort study (Prostate Biopsy Effects: ProBE) nested within Prostate Testing for Cancer and Treatment (ProtecT) study.

Participants Between 1999 and 2008, 227000 community dwelling men aged 5069 years were identified at 352 practices and invited to counselling about PSA testing. 111148 attended a nurse led clinic in the community, and 10297 with PSA concentrations of $3-20 \mathrm{ng} / \mathrm{mL}$ were offered biopsy within ProtecT. Between February 2006 and May 2008, 1147/1753 (65\%) eligible men (mean age 62.1 years, mean PSA 5.4 $\mathrm{ng} / \mathrm{mL}$ ) having 10 core transrectal ultrasound guided biopsy under antibiotic cover in the context of ProtecT were recruited to the ProBE study.

Outcome measures Purpose designed questionnaire administered at biopsy and 7 and 35 days after the procedure to measure frequency and effect of symptoms related to pain, infection, and bleeding; patients' attitude to repeat biopsy assessed immediately after biopsy and 7 days later; participants' healthcare resource use within 35 days of biopsy
\end{abstract}

evaluated by questionnaire, telephone follow-up, and medical note review; each man's adverse event profile graded according to symptoms and healthcare use.

Results Pain was reported by $429 / 984$ (43.6\%), fever by $172 / 985$ (17.5\%), haematuria by $642 / 976(65.8 \%)$, haematochezia by $356 / 967$ (36.8\%), and haemoejaculate by $605 / 653$ (92.6\%) men during the 35 days after biopsy. Fewer men rated these symptoms as a major/moderate problem-71/977 (7.3\%) for pain, 54/981 (5.5\%) for fever, 59/958 (6.2\%) for haematuria, 24/951 (2.5\%) for haematochezia, and 172/646 (26.6\%) for haemoejaculate. Immediately after biopsy, 124/1142 (10.9\%, 95\% confidence interval 9.2 to 12.8 ) men reported that they would consider further biopsy a major or moderate problem: seven days after biopsy, this proportion had increased to $213 / 1085$ (19.6\%, $17.4 \%$ to $22.1 \%$ ). A negative attitude to repeat biopsy was associated with unfavourable experience after the first biopsy, particularly pain at biopsy (odds ratio $8.2, \mathrm{P}<0.001)$ and symptoms related to infection $(7.9, \mathrm{P}<0.001)$ and bleeding (4.2, $\mathrm{P}<0.001)$; differences were evident between centres $(\mathrm{P}<0.001) .119 / 1147$ (10.4\%, 8.7\% to 12.3\%) men reported consultation with a healthcare professional (usually their general practitioner), most commonly for infective symptoms. Complete data for all index symptoms at all time points were available in 851 participants. Symptoms and healthcare use could be used to grade these men as follows: grade 0 (no symptoms/contact) $18(2.1 \%, 1.3 \%$ to $3.3 \%)$; grade 1 (minor problem/no contact) $550(64.6 \%, 61.4 \%$ to $67.8 \%)$; grade 2 
(moderate/major problem or contact) 271 (31.8\%, 28.8\% to $35.1 \%$ ); grade 3 (hospital admission) 12 (1.4\%, $0.8 \%$ to $2.4 \%$ ); and grade 4 (death) 0 . Grade of adverse event was associated with an unfavourable attitude to repeat biopsy (Kendall's $\tau$-b ordinal by ordinal $0.29, \mathrm{P}<0.001$ ).

Conclusion This study with a high response rate of $89 \%$ at 35 days in men undergoing biopsy in the context of a randomised controlled trial has shown that although prostate biopsy is well tolerated by most men, it is associated with significant symptoms in a minority and affects attitudes to repeat biopsy and primary care resource use. These findings will inform men who seek PSA testing for detection of prostate cancer and assist their physicians during counselling about the potential risks and effect of biopsy. Variability in the adverse event profile between centres suggests that patients' outcomes could be improved and healthcare use reduced with more effective administration of local anaesthetic and antibiotics.

Trial registration Current Controlled Trials ISRCTN20141297.

\section{Introduction}

Prostate cancer is a major health problem worldwide; 899000 new cases were diagnosed in 2008 (13.6\% of total incident cancers), and an estimated 258000 deaths (6.1\% of cancer deaths) from the disease occurred. ${ }^{1}$ Recent evidence from large European randomised studies of screening suggests a reduction in disease specific mortality ranging between $20 \%$ and $44 \%,{ }^{23}$ but at the cost of over-detection and potential overtreatment, ${ }^{4}$ which precludes the introduction of screening as public health policy. While the debate continues, men who seek early detection of prostate cancer by testing for prostate specific antigen (PSA) need accurate information on the consequences of the diagnostic process as well as the therapeutic dilemma. In men who have PSA testing, prostate biopsy is needed for a histological diagnosis of prostate cancer. Whereas considerable attention has been paid to PSA testing, relatively little is known about the effect of prostate biopsy in this group of otherwise healthy men. One of the criteria for suitability of a test for screening is that it is acceptable to the population being screened. In the context of prostate cancer, this criterion is often applied only to PSA testing but is also relevant in the context of subsequent prostate biopsy to provide the definitive diagnosis. Laboratories carrying out PSA testing for the National Health Service are subject to quality assurance under the United Kingdom National External Quality Assurance Service. Comparatively, transrectal ultrasound guided prostate biopsy (TRUS-Bx), the most commonly used technique for prostate biopsy in these asymptomatic men, has received little attention and is not subject to any similar external quality assurance.

TRUS-Bx was introduced more than two decades ago, initially without any form of analgesia, for the detection of prostate cancer. ${ }^{5}$ The procedure has evolved, primarily to improve the rate of cancer detection by increasing the number of biopsy cores taken. ${ }^{6}$ To reduce morbidity and improve tolerability of increasing the number of cores, the use of prophylactic antibiotics and analgesia in the form of intrarectal lidocaine gel or periprostatic infiltration of local anaesthetic have become commonplace, with considerable variability in practice and outcomes. ${ }^{789}$ TRUS-Bx can be associated with appreciable morbidity, including sepsis, pain, bleeding, and even death on rare occasions. The true effect of biopsy is difficult to ascertain from the literature - the reported morbidity is highly variable, and although post-biopsy adverse events are often referred to as "major" or "minor," the basis of this classification is arbitrary and inconsistent. The incidence of major complications in the Rotterdam cohort of the European Randomized study of Screening for Prostate Cancer (ERSPC) was $12 \%$ (7.5\% pain, $4 \%$ fever, $0.5 \%$ hospital admission for sepsis, $0.4 \%$ admission for acute urinary retention),${ }^{10}$ in contrast with a reported incidence of "major" complications of $0.1 \%$ in an Austrian screened population $(n=1051) .{ }^{11}$ A systematic review of TRUS-Bx observed that only 41 of 87 studies made any mention of adverse events, and where mention was made no standardised approach to reporting was used. ${ }^{12}$ Furthermore, the acceptability of re-biopsy is uncertain: in the era preceding widespread use of local anaesthetic, a British study reported that $19 \%$ of men refused re-biopsy without general anaesthesia, ${ }^{13}$ and the Finnish study of screening for prostate cancer observed that $18 \%$ of screened men would not accept a repeat biopsy. ${ }^{14}$ In contrast, Djavan et al reported that of 820 Austrian men previously biopsied, all re-attended for re-biopsy as an outpatient when invited. ${ }^{11}$ The factors influencing this attitude towards repeat biopsy remain uncertain, but it is important as around two thirds of men initially have a negative biopsy and may need another. ${ }^{15}$

The wide variability in adverse events and acceptability probably reflects a combination of expertise, patients' expectations, and inconsistent reporting. Little evidence based information about the experience of TRUS-Bx is available to share with men having the procedure. Additionally, no systematic investigation of the acceptability of TRUS-Bx and association between adverse events after TRUS-Bx and attitude to re-biopsy in a PSA-tested population has been done. The aims of this study were to measure the effect of adverse events after a first prostate biopsy in asymptomatic men having PSA testing; to assess early attitudes to repeat biopsy; to estimate the effect of adverse events from TRUS-Bx on healthcare resource use; and to develop a simple classification system for consistent reporting of adverse events after prostate biopsy.

\section{Methods \\ Design and sample size}

A prospective cohort study (ProBE-Prostate Biopsy Effects) was embedded in the ongoing Prostate Testing for Cancer and Treatment (ProtecT) study, which is a large multicentre randomised controlled trial started some years previously to evaluate the effectiveness, cost effectiveness, and acceptability of treatments for men with localised prostate cancer. ${ }^{15}{ }^{16}$ General medical practices in and around nine cities throughout the United Kingdom recruited men to the ProtecT study. Lead partners of the practices received letters of invitation for their practice to participate in the study, detailing the rationale for a randomised controlled trial of treatment in clinically localised prostate cancer detected by PSA. Participating practices in turn sent letters inviting men aged 50-69 years registered at the practice to attend for PSA counselling. Nurse led clinics were held in a primary care setting, where participants with an estimated life expectancy of a minimum of 10 years and without important cardiorespiratory comorbidity were given detailed information about the implications of PSA testing, the uncertainties about treatments, and the need for a treatment trial; consent was sought thereafter for PSA testing. Biopsy was offered to all men with a PSA concentration of $3.0 \mathrm{ng} / \mathrm{mL}$ or above. The sample size calculation suggested that 880 participants would provide a precise estimate of an expected hospital admission rate of $1 \%,{ }^{10}$ with a $95 \%$ confidence interval of 0.4 to $2 \%$. The target was to enrol 1200 men, allowing a priori for a $25 \%$ dropout rate.

\section{Population and exposure}

In February 2006, the ProBE study started recruitment in eight of the nine ProtecT centres. Men presenting with a PSA concentration of 3.0-19.9 ng/mL, with no previous experience of TRUS-Bx, were invited to participate in ProBE in addition 
to their involvement in the ProtecT study. As many practices were part way through recruitment to ProtecT when ProBE started and stopped recruitment, not all practices nor all men at any given practice were offered the study.

The intention was for the ProBE study to recruit consecutive men attending for a prostate biopsy in ProtecT from February 2006 until the sample size of 1200 was reached (see above). Resource limitation at the height of recruitment to ProtecT meant that some biopsy sessions were not covered by research staff, so not all men attending biopsy sessions were offered participation in ProBE.

All men invited to join the ProBE study were given patients' information sheets on ProBE as well as ProtecT, in addition to local instructions related to the biopsy procedure. Baseline data including weight, height, drug history, comorbidity, Hospital Anxiety and Depression Scale (HADS) score, and urinary, bowel, and sexual symptoms were assessed by using the validated International Continence Society-male, International Consultation on Incontinence Modular Questionnaire-urinary incontinence, and University of California, Los Angeles Prostate Cancer Index questionnaires. ${ }^{17} 1819$ Outpatient TRUS-Bx was carried out in the left lateral decubitus position under antibiotic cover by using a 10 core lateral biopsy template. ${ }^{20}{ }^{21}$ In a pre-study questionnaire, seven of the eight centres reported using periprostatic infiltration of local anaesthetic ( $1 \%$ or $2 \%$ lidocaine) routinely before biopsy. All men received antibiotic prophylaxis according to contemporary practice; inter-centre variability existed, but each centre used a consistent schedule throughout the study period. A midstream specimen of urine was sent for culture immediately before antibiotic administration. Coumarin anticoagulant and clopidogrel treatment were discontinued up to 10 days before biopsy and advice sought as to appropriate substitutes if indicated. Aspirin was continued at the discretion of the physician doing the biopsy. Men were kept under observation after the biopsy until they voided. Urine was assessed for haematuria according to a four point colorimetric scale. Each centre provided its own post-biopsy written instructions and contact details.

\section{Outcome measures}

\section{Symptoms}

Men self reported pain and discomfort (referred to as pain hereafter) immediately after and seven days after TRUS-Bx on a four point Likert-type scale as none, mild, moderate, or severe. Specific related complications such as fever, flu-like shivers, pain, haematuria, haematochezia, and haemoejaculate were self reported at seven and 35 days after TRUS-Bx as absent or present following biopsy on a purpose designed questionnaire (TRUS-BxQ; see appendix 1). This included the validated International Continence Society-male, International Consultation on Incontinence Modular Questionnaire - urinary incontinence, and University of California, Los Angeles Prostate Cancer Index questionnaires. ${ }^{17} 1819$ For each reported symptom, participants were asked to grade the degree of "problem" associated with its presence as none, minor, moderate, or major. We used this information to derive a binary outcome for each symptom, as present with moderate/severe problem versus not present/minor problem. During the pilot phase (February 2006 to April 2006), an expert committee (appendix 2) reviewed the initial data from the TRUS-BxQ to optimise face validity and content validity.

\section{Attitude to re-biopsy}

To assess attitudes to possible further biopsies immediately after the biopsy and seven days later, men were asked to record on a four point Likert-type scale (no problem, minor, moderate, major problem) "how much of a problem would you find having another biopsy in the future?" Analyses explored the relations between men's reports of pain and symptoms and their attitude to re-biopsy.

\section{Healthcare resource use}

Men reported any contact with healthcare services in the TRUS-BxQ at seven and 35 days after biopsy. A non-clinical research assistant did semi-structured telephone interviews with men between seven and 10 days after biopsy. When self reported data were unclear or missing, we searched medical records (written and electronic) for any episode of contact with any provider in primary or secondary care occurring within 35 days of the date of biopsy. We used data from the participants' interviews, TRUS-BxQ, and the review of hospital records to ascertain the reason for any hospital admission. When contact with primary care was detected or reported, we made an assessment of its relevance to the biopsy procedure, on the basis of a free text description provided by participants in the self completed TRUS-BxQ and review of the clinical records where necessary.

\section{Adverse event classification}

We grouped adverse events of fever, flu-like shivers, haematuria, haematochezia, and haemoejaculate together as "infective," "haemorrhagic," and combined "infective/haemorrhagic" symptoms. We classified adverse events in a manner consistent with the National Cancer Institute's common toxicity criteria version 3.0 as grade $0=$ no adverse event reported and no biopsy related healthcare contact, grade $1=$ symptoms reported but causing no/minor problem and no biopsy related healthcare contact, grade 2 = symptom reported causing major/moderate problem or biopsy related healthcare contact without hospital admission, grade $3=$ hospital admission within 35 days of biopsy, and grade $4=$ death within 35 days of biopsy. ${ }^{22}$ This analysis included only men with a complete dataset of symptoms at both assessments.

\section{Data analysis}

We used SPSS statistical software version 18.0 to do data analyses using the available data; we did not impute any missing data. We present the proportion of men who experienced each outcome, with $95 \%$ confidence intervals calculated by using Wilson's method (CIA software, version 2.2.0, www.som. southampton.ac.uk/cia). We estimated associations between risk factors and binary outcome measures (for example, moderate/major problem with re-biopsy, healthcare contact, hospital admission) as odds ratios by using logistic regression with adjustment for age and centre. For clarity, where the symptom scale is reduced to a binary distinction, this distinguishes men reporting a moderate/major problem from those reporting a mild/no problem. We used the logistic regression models as the basis of likelihood ratio tests to calculate $\mathrm{P}$ values. We adjusted for age and recruitment centre by adding dummy variables to the regression models - three dummy variables distinguishing four age groups (50-54, 55-59, 60-64, and 65-69 years) and seven dummy variables distinguishing eight study centres. 


\section{Results}

In the ProtecT study overall, of the 227000 men aged 5069 years who were identified at 352 practices and invited to nurse led clinics in the community for counselling about PSA testing, 111148 attended. Of the 10297 men offered TRUS-Bx within ProtecT, 1753 attended for biopsy between February 2006 and May 2008 and were eligible to enter the ProBE study; 1147 (65\%) consented to participation. Table $1 \Downarrow$ summarises baseline data.

Of the 1147 men who had TRUS-Bx within ProBE, TRUS-BxQ data were available for $1144(99.7 \%)$ at baseline, $1090(95.0 \%)$ at seven days, and 1018 (88.8\%) at 35 days post-biopsy. Telephone follow-up, primary care, and hospital record data were available for all 1147 participants. We found no difference between the men recruited in the eight centres with regard to age $(\mathrm{P}=0.086)$, PSA concentration $(\mathrm{P}=0.51)$, body mass index $(\mathrm{P}=0.54)$, or HADS anxiety $(\mathrm{P}=0.83)$ or depression scores $(\mathrm{P}=0.2)$. A difference in mean prostate volume existed $(\mathrm{P}<0.001)$.

\section{Symptoms, degree of associated problem, and duration}

Immediately after biopsy, 37/1134 (3.3\%, 95\% confidence interval $2.4 \%$ to $4.5 \%$ ) men reported light-headedness and three $(0.3 \%, 0.1 \%$ to $0.8 \%)$ experienced a short period of syncope. All men voided after the procedure. Moderate or severe haematuria was present in 78/1055 (7.4\%, 6.0\% to $9.1 \%)$ post-biopsy urine samples and was associated with large blood clots in 27 (2.6\%, $1.8 \%$ to $3.8 \%)$. Three additional men passed clots without moderate or severe haematuria.

Table $2 \Downarrow$ shows the number of men who reported pain, fever, flu-like shivers, haematuria, haematochezia, and haemoejaculate within seven and 35 days of biopsy and the proportion who reported a moderate/major problem associated with each symptom. Approximately one third of men $(n=340 / 1147)$ reported no sexual activity within 35 days after biopsy, and the prevalence of haemoejaculate is therefore limited to those who were sexually active during the study period. Although the prevalence of each symptom and combination of symptoms was relatively high, most men reported the presence of a symptom as being no problem or a minor problem (table $2 \Downarrow$ and box).

\section{Attitude to repeat biopsy}

Immediately after the procedure, $124 / 1142(10.9 \%, 9.2 \%$ to $12.8 \%$ ) men reported that having a repeat biopsy would represent a moderate/major problem. This rose to 213/1085 (19.6\%, 17.4\% to $22.1 \%$ ) when the question was repeated seven days later ( $\mathrm{P}=0.001$, McNemar's test for a null hypothesis of no difference in the proportion finding re-biopsy a moderate/major problem). The proportion of men considering repeat biopsy to be a moderate/major problem differed by centre at both time points $(\mathrm{P}<0.001)($ table $3 \Downarrow)$. These differences persisted following the exclusion of centre $3(\mathrm{P}=0.004)$, where local anaesthetic was not administered routinely.

A strong association existed between an unfavourable attitude to repeat biopsy at seven days and pain reported at both time points (immediately post-biopsy: odds ratio $12.1, \mathrm{P}<0.001$; at seven days: odds ratio $8.2, \mathrm{P}<0.001)$ as well as symptoms within seven days related to infection (odds ratio $7.89, \mathrm{P}<0.001$ ) and bleeding $(4.24, \mathrm{P}<0.001)($ table $4 \Downarrow)$.

\section{Healthcare contact}

No deaths occurred in this cohort within 35 days of biopsy ( $95 \%$ confidence interval 0 to $0.4 \%)$. In that period, $15 / 1147(1.3 \%$, $0.8 \%$ to $2.1 \%$ ) men needed hospital admission for sepsis $(n=7)$, urinary retention $(n=3)$, haematuria $(n=2)$, rectal bleeding $(n=1)$, or other diagnoses $(n=2)$. All admissions due to sepsis occurred within three days of biopsy, whereas one man with retention was admitted three weeks after biopsy. A further $119(10.4 \%$, $8.7 \%$ to $12.3 \%$ ) men initiated a biopsy related consultation with their general practitioner $(n=92)$, urology department nurse (14), or other source of medical advice (13) such as NHS Direct. The predominant reasons for seeking healthcare advice in primary care were infective symptoms $(\mathrm{n}=38)$, urinary symptoms including haematuria (34), haemoejaculate (14), possibility of antibiotic related adverse events such as diarrhoea or a skin rash (14), and discomfort/bleeding on defecation (10). We found no evidence that such contacts varied across centres $(\mathrm{P}=0.73)$.

Table $4 \Downarrow$ lists selected variables examined for associations with healthcare contact within 35 days. Biopsy related healthcare contact within 35 days was more likely in men with a previous history of urinary tract infection $(\mathrm{P}=0.036)$ compared with others, and in men who reported moderate/severe pain immediately after the procedure $(\mathrm{P}=0.017)$. Cross sectional analysis showed a strong association between healthcare contact and symptoms of infection, bleeding, or both at seven days $(\mathrm{P}<0.001)$.

Owing to the low number of events, we could investigate only crude associations with hospital admissions. We found evidence of a higher risk of hospital admission in men treated with non-steroidal agents $(3 / 58(5 \%) v 11 / 1035(1.1 \%), \mathrm{P}=0.037)$ and those reporting a moderate/severe problem with symptoms of infection $(7 / 47(15 \%) v 7 / 1036(0.7 \%), \mathrm{P}<0.001)$ or bleeding $(6 / 179(3 \%) \vee 9 / 843(1.1 \%), \mathrm{P}=0.039)$ at 7 days. None of the men with a history of prostatitis $(\mathrm{n}=18)$ or warfarin treatment $(n=14)$ or who had an infected midstream urine sample before biopsy $(n=18)$ were admitted to hospital within 35 days of the procedure.

\section{Adverse event classification}

Complete data on the five index symptoms at seven and 35 days were available for 851/1147 (74.2\%) men. We classified adverse events as grade 0 (no symptoms/contact) in $18(2.1 \%, 1.3 \%$ to $3.3 \%$ ), grade 1 (minor) in $550(64.6 \%, 61.4 \%$ to $67.8 \%)$, grade 2 (moderate/major) in $271(31.8 \%, 28.8 \%$ to $35.1 \%)$, and grade 3 (inpatient/hospital admission) in 12 (1.4\%, $0.8 \%$ to $2.4 \%$ ); no participants had a grade 4 event (death). We found strong evidence of an association between the grade of adverse event at 35 days and an unfavourable attitude to repeat biopsy at seven days (Kendall's $\tau$-b ordinal by ordinal 0.29, $\mathrm{P}<0.001$ ).

\section{Exploratory analyses}

\section{Prostate volume, age, and attitude to repeat biopsy}

We found weak evidence that older men were more likely to report a favourable attitude to repeat biopsy than were younger participants (odds ratio per 5 year increase in age between 50 and 69 years: $0.86,0.75$ to $1.00, \mathrm{P}=0.056)\left(\right.$ table $\left.4^{4}\right)$. Men who considered repeat biopsy to be a moderate/major problem were more likely to have a smaller prostate volume $(\mathrm{P}=0.027)$ (table $4 \Downarrow)$. 


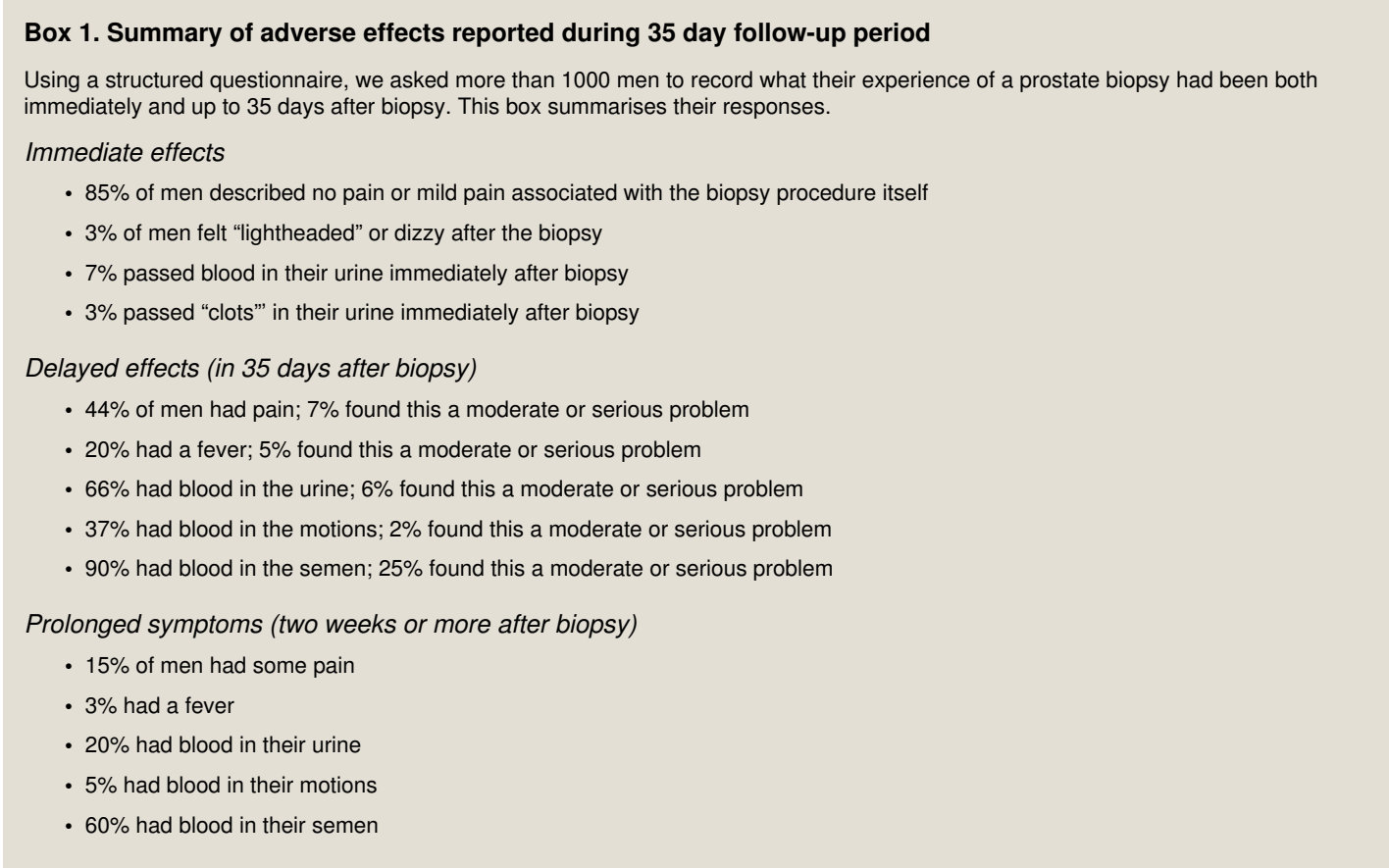

\section{Prostate cancer diagnosis and healthcare contact}

Prostate cancer was diagnosed on biopsy in 406/1147 (35.4\%, $32.6 \%$ to $38.2 \%$ ) men. We found a difference in cancer detection rates ranging between $23 \%$ (14\% to $36 \%$ ) and $53 \%$ (40\% to $65 \%)$ across the eight centres $(\mathrm{P}=0.008)$. We found no convincing evidence that men subsequently diagnosed as having cancer were more likely to come into contact with healthcare services for a biopsy related adverse event within 35 days of the procedure compared with men in whom cancer was not diagnosed (48/406 (11.8\%) v 87/741 (11.6\%); odds ratio 1.03, 0.70 to $1.50, \mathrm{P}=0.89$, adjusted for age and study centre). The risk of hospital admission was $1.7 \%$ in men subsequently diagnosed as having cancer and $1.1 \%$ in those without cancer $(\mathrm{P}=0.84)$.

\section{Discussion}

This multicentre prospective study with a high response rate of $89 \%$ at 35 days provides generalisable quantitative data about the events occurring in the 35 days after a first prostate biopsy. The findings are based on patient reported outcomes and verifiable criteria, including healthcare resource use. Whereas prostate biopsy was reasonably well tolerated in most men, a few men rated post-biopsy pain and infective/haemorrhagic symptoms as a major/moderate problem- $7.3 \%$ for pain, $5.5 \%$ for fever, $6.2 \%$ for haematuria, $2.5 \%$ for haematochezia, and $26.6 \%$ for haemoejaculate. Immediately after biopsy, $10.9 \%$ reported that they would consider further biopsy a major or moderate problem; seven days after biopsy, this proportion had increased to $19.6 \%$. A negative attitude to repeat biopsy was associated with unfavourable experience after the first biopsy, particularly pain at biopsy; differences were evident between centres carrying out the biopsies. Consultation with a healthcare professional (usually their general practitioner) was reported by $10.4 \%$, most commonly for infective symptoms.

Transrectal ultrasound guided biopsy of the prostate is an ambulatory procedure commonly carried out in consulting rooms and outpatient and radiology departments in small and large hospitals. Although accurate figures for the number of procedures carried out in the UK each year are difficult to elicit, approximately 37000 new cases of prostate cancer are diagnosed annually (http://info.cancerresearchuk.org/cancerstats/types/ prostate), with a cancer detection rate at biopsy of around $35 \% .{ }^{17}$ Assuming that around 100000 procedures are carried out every year in the UK alone would thus be reasonable.

The results of this study are particularly relevant to screening for prostate cancer rather than the performance of prostate biopsy for confirmation of a diagnosis in clinically evident disease. The cohort is drawn from asymptomatic men, invited for PSA testing in the context of a large randomised controlled trial (ProtecT), with a first biopsy at a PSA concentration of 3.0-19.9 $\mathrm{ng} / \mathrm{mL}$, a range commonly held to be appropriate for detection of localised prostate cancer suitable for treatment with curative intent. None of the men had repeat biopsy during the timeframe specified. Particular emphasis was placed on the perception of the man having the biopsy, as well as the identification of adverse events. This emphasis allowed the study to measure how a man perceives the degree of "problem" associated with individual symptoms resulting from a first prostate biopsy. Cross referencing these perceptions with healthcare resource use provided useful triangulation. Maintaining a high response rate of $95 \%$ at seven days and $89 \%$ at 35 days is a particular strength of the study.

A significant number of men experienced difficulties during or after the biopsy, primarily associated with pain, sepsis, or bleeding. Very few men experienced no symptoms at all $(2.1 \%$, grade 0 ) in the five weeks after biopsy. Of those who did experience one or more symptoms, most considered them to be of little consequence (64.6\%, grade 1$)$. The remaining third experienced adverse events that they considered to be a moderate or severe problem. Of interest, the presence of blood in a man's ejaculate (haemoejaculate) after biopsy is seldom reported in the literature and is often labelled as a "minor" adverse event. In this study, haemoejaculate was perceived as a moderate to severe problem for around one quarter of sexually active men (table $2 \Downarrow$ ). Further investigation of the problems generated by this symptom is warranted, and information about its duration and persistence should be given to all men having biopsies.

During initial counselling before PSA testing, men are informed of the possible requirement for repeat biopsies beyond the first procedure, in the event of equivocal results or a benign biopsy 
with persistent risk factors such as a persistently raised serum PSA concentration. A recent study from the United States reported that $38 \%$ of men having a first biopsy will have a repeat procedure within five years of the initial biopsy; the proportion reaches $44 \%$ in men under the age of 70 years. ${ }^{23}$ Several studies have shown that repeat biopsy leads to a detection rate for prostate cancer of $19-59 \%,{ }^{24} 2526$ so understanding how a first biopsy affects a man's attitude to additional procedures if needed is essential. In this study, $11 \%$ and $20 \%$ of men reported an unfavourable attitude to repeat biopsy immediately after biopsy and seven days later; pain experienced at biopsy and infective symptoms in the week after biopsy were strongly associated with this response. Systematic TRUS-Bx was introduced initially without local anaesthetic, although this has been adopted in clinical practice more recently. ${ }^{59} 927$ Meta-analyses have described a significant reduction in pain scores with periprostatic nerve blocks ${ }^{28} 29$; doubt remains, however, as to whether the reduction in pain scores translates to clinically meaningful changes, particularly as infiltration of local anaesthetic itself has been shown to be associated with pain. ${ }^{30}$ The association between levels of pain experienced and attitude to having repeat biopsy described in this study is an important observation that supports the mandatory use of periprostatic nerve blockade with local anaesthetic. Even among centres where local anaesthetic was routinely administered, inter-centre variability in its effect existed. This suggests that the technique of administration may need to be standardised to ensure effectiveness of local anaesthesia.

As well as resulting in a negative attitude to re-biopsy, infective complications were the most common reason for seeking medical advice or primary care intervention (10.4\%). Most symptoms were evident within seven days of biopsy, with a small proportion of men continuing to report symptoms for up to five weeks after biopsy (box). This information is particularly valuable for general practitioners when counselling patients before PSA testing. The American Urological Association's best practice policy statement recommends administration of a fluoroquinolone as uniform antimicrobial prophylaxis in all men having TRUS-Bx. ${ }^{31}$ Current practice, however, has been influenced predominantly by randomised controlled trials indicating the efficacy of a single dose of ciprofloxacin in reducing the incidence of infective complications after TRUS-Bx ${ }^{32} 33$; subsequent studies have suggested that prolonging treatment to three days results in superior clinical effectiveness. ${ }^{34}$ A recent report has identified an increasing rate of sepsis related admission to hospital after prostate biopsy in the decade between $1996(0.6 \%)$ and $2005(3.6 \%) .^{35}$ This may be related to a general reduction in peri-biopsy antibiotic use, ${ }^{36}$ but the development of quinolone resistance may also be implicated. ${ }^{37} 383940$ Against this backdrop, antibiotic prophylaxis for TRUS-Bx is universally used, but without consensus on best practice. ${ }^{41}$ We have shown some evidence of differences in infective complications between centres, but our study was not specifically designed to evaluate the effectiveness of antibiotic prophylaxis; this warrants further prospective investigation.

The classification of adverse events described in this study is similar to that described after systemic chemotherapy. It is based on a combination of reported events and their effect from a patient's perspective. Its wider use needs further validation and is likely to show the need to standardise and refine the technique of prostate biopsy to minimise its adverse events. No fatal events occurred in our cohort. This is not surprising, as our participants were relatively healthy with an upper age limit of 69 years and no symptoms. Mortality after prostate biopsy has been reported as ranging between $0.09 \%$ and $1.3 \%$ at 120 days,${ }^{35} 42$ and it is associated with older, less healthy men.

In contrast with a large retrospective Canadian study referred to earlier, ${ }^{35}$ we found no difference in our prospective study in hospital admission rate (grade 3 adverse event) between participants with cancer and those without (1.7\% v 1.1\%). The findings of the Canadian study may result from the investigation of a population at different risk or, as acknowledged by the authors, from inaccuracy in coding associated with its retrospective nature.

\section{Limitations of study}

The ProBE study cohort included asymptomatic men aged between 50 and 69 years presenting for a first prostate biopsy after a PSA test, received through the ProtecT study during the period February 2006 to May 2008. The ProBE study was carried out over this limited period, sampling approximately $11 \%$ of the ProtecT participants. The cohort did not include men seen in routine UK clinical practice with urinary symptoms or clinically suspected prostate cancer. Of the men eligible for inclusion, $65 \%$ were recruited and thus are likely to be representative of the men who attended for PSA testing within the ProtecT study. Embedding the ProBE study in the ProtecT trial was beneficial, providing a standardised biopsy template and recruitment from a wide range of practices across the UK and improving compliance with follow-up. However, participants had responded to a single written invitation to attend for PSA testing sent out via general practices, so non-responders may not be represented in this study. The observations are likely to remain valid in the context of men seeking PSA testing for detection of prostate cancer.

In the absence of nationally agreed, evidence based patient information leaflets, each centre delivered its own information about the biopsy process, which may have influenced men's views. Recall bias may also have occurred for recording the duration of symptoms, as men were asked to summarise their experience over the previous four weeks in the 35 day questionnaire. For the main analyses, we required data with evaluable responses from both the seven day and 35 day questionnaire assessments. This reduced the numbers and may have led to some degree of underestimation of the prevalence of adverse events. Although relevant to the overall outcome, some of the reported symptoms (such as fever/shivers) may not have been related to the biopsy itself and may have inadvertently influenced a negative attitude to repeat biopsy.

\section{Additional items}

Administration of local anaesthesia is important in determining pain experienced, but other factors, such as environment, training, education, and nursing care, will clearly affect anxiety and pain. We have explored some of these factors in a linked qualitative study (data not included). Similarly, although we have sought men's initial and early views on the acceptability of repeat biopsy, attitudes may change with time; we shall therefore follow up this cohort carefully to analyse the re-biopsy rate and assess patients' subsequent views.

While healthcare providers await further evidence to decide whether screening for prostate cancer should become public health policy, primary care physicians need to be well informed of the risks and adverse events related to TRUS-Bx as well as PSA before testing. The findings of this study contribute further generalisable quantitative data about the potential harms associated with making a diagnosis of prostate cancer in asymptomatic men. 


\section{Generalisability}

The ProBE study investigated a large cohort of men aged between 50 and 69 years who had TRUS-Bx for the first time as a result of a PSA concentration between 3.0 and $20 \mathrm{ng} / \mathrm{mL}$. The cohort comprised $65 \%$ of eligible men biopsied within a trial population. The results are likely to be generalisable to all asymptomatic men in this age group who seek diagnosis of prostate cancer through PSA testing and in the context of screening. They may, however, not be applicable to younger or older men, those with clinical evidence of prostate cancer, or men who have previously had prostate biopsy.

\section{Conclusions}

After prostate biopsy, one third of men having first time TRUS-Bx for a high PSA reported moderate to severe biopsy related symptoms. Immediately after TRUS-Bx, one in 10 men had an unfavourable attitude to repeat biopsy, rising to one in five later. This was associated with adverse events in the seven days after biopsy and seemed to affect younger men more; as these men are most likely to benefit from early diagnosis of prostate cancer as well as require re-biopsy, this requires further investigation. Within 35 days of biopsy, $1.3 \%$ of men required admission to hospital and a further $119(10.4 \%)$ men initiated a biopsy related consultation with their general practitioner $(n=92)$, urology department nurse $(n=14)$, or other source of medical advice $(n=13)$ such as NHS Direct, most commonly for infective symptoms. The adverse event classification scheme described may help to show variation in rates of adverse events across centres that arise from differences in information and biopsy technique. Training, protocols, and consistent reporting methods should be considered to achieve excellence in practice. Information for patients about biopsy should be standardised and include the findings of this study, so that men and physicians are fully informed about the risks and benefits of the diagnostic process before they embark on prostate cancer detection.

The ProBE (Prostate Biopsy Effects) Study Group: Prasad Bollina, Sue Bonnington, Lynn Bradshaw, Debbie Cooper, Liz Down, Alan Doherty, Garrett Durkan, Emma Elliott, David Gillatt, Pippa Herbert, Peter Holding, Joanne Howson, Mandy Jones, Roger Kockelbergh, Howard Kynaston, Teresa Lennon, Norma Lyons, Hing Leung, Malcolm Mason, Hilary Moody, James N'Dow, Philip Powell, Alan Paul, Stephen Prescott, Patricia O'Sullivan, Pauline Thompson, and Sarah Tidball.

Contributors: DJR, JAL, and FCH devised the protocol and arranged integration into the ongoing ProtecT study. DJR and JAL contributed equally to the overall project and the final manuscript and were primarily responsible for the completed manuscript. CM contributed to study design, provided statistical expertise, directly contributed to data analysis, and helped to interpret the analysis. JLD contributed to study design and particularly advised on questionnaire validation. $A D$ contributed to study design and acted as medical consultant to the study. LG contributed to study design, carried out the validation of the TRUS-Bx questionnaire, and acted as nurse consultant to the study. MD contributed to study design and dealt with data queries. JWFC acted as medical consultant to the study. KA carried out the preliminary qualitative study that highlighted areas of concern and contributed to study design. DEN advised on research priorities, advised on integration into the ongoing ProtecT study, and contributed to study design. All authors critically revised the manuscript and revised it for important intellectual content. DJR and JAL are the guarantors.

Funding: The ProBE study was funded by the Prostate Cancer Risk Management Group. The study and authors are independent of the funding body. The ProtecT study is funded by NIHR HTA.
Competing interests: All authors have completed the Unified Competing Interest form at www.icmje.org/coi_disclosure.pdf (available on request from the corresponding author) and declare: no support from any organisation for the submitted work; no financial relationships with any organisations that might have an interest in the submitted work in the previous three years; and no other relationships or activities that could appear to have influenced the submitted work.

Ethical approval: The ProBE study received approval from Trent MREC as part of the ProtecT (Prostate Testing for Cancer and Treatment) trial (ISRCTN20141297).

Data sharing: No additional data available for sharing.

1 Ferlay J, Shin HR, Bray F, Mathers C, Parkin DM. GLOBOCAN 2008, Cancer incidence and mortality worldwide: IARC CancerBase no 10. International Agency for Research on Cancer, 2010

2 Schroder FH, Hugosson J, Roobol MJ, Tammela TL, Ciatto S, Nelen V, et al. Screening and prostate-cancer mortality in a randomized European study. N Engl J Med 2009;360:1320-8.

3 Hugosson J, Carlsson S, Aus G, Bergdahl S, Khatami A, Lodding P, et al. Mortality results from the Goteborg randomised population-based prostate-cancer screening trial. Lancet Oncol 2010;11:725-32.

4 Djulbegovic M, Beyth RJ, Neuberger MM, Stoffs TL, Vieweg J, Djulbegovic B, et al. Screening for prostate cancer: systematic review and meta-analysis of randomised controlled trials. BMJ 2010;341:c4543.

5 Hodge KK, MCNeal JE, Terris MK, Stamey TA. Random systematic versus directed ultrasound guided transrectal core biopsies of the prostate. J Urol 1974;142:71-4.

6 Patel AR, Jones JS. Optimal biopsy strategies for the diagnosis and staging of prostate cancer. Curr Opin Urol 2009;19:232-7.

Loeb S. Prostate biopsy: a risk-benefit analysis. J Urol 2010;183:852-3.

8 Lee G, Attar K, Laniado M, Karim O. Trans-rectal ultrasound guided biopsy of the prostate: nationwide diversity in practice and training in the United Kingdom. Int Urol Nephrol 2007;39:185-8.

9 Leippold T, Preusser S, Engeler D, Inhelder F, Schmid HP. Prostate biopsy in Switzerland: a representative survey on how Swiss urologists do it. Scand J Urol Nephrol 2008;42:18-23.

10 Raaijmakers R, Kirkels WJ, Roobol MJ, Wildhagen MF, Schroder FH. Complication rates and risk factors of 5802 transrectal ultrasound-guided sextant biopsies of the prostate within a population-based screening program. Urology 2002;60:826-30.

11 Djavan B, Waldert M, Zlotta A, Dobronski P, Seitz C, Remzi M, et al. Safety and morbidity of first and repeat transrectal ultrasound guided prostate needle biopsies: results of a prospective European prostate cancer detection study. J Urol 2001;166:856-60.

12 Eichler K, Hempel S, Wilby J, Myers L, Bachmann LM, Kleijnen J. Diagnostic value of systematic biopsy methods in the investigation of prostate cancer: a systematic review. J Urol 2006;175:1605-12.

13 Irani J, Fournier F, Bon D, Gremmo E, Dore B, Aubert J. Patient tolerance of transrectal ultrasound-guided biopsy of the prostate. Br J Urol 1997;79:608-10.

14 Mkinen T, Auvinen A, Hakama M, Stenman UH, Tammela TL. Acceptability and complications of prostate biopsy in population-based PSA screening versus routine clinical practice: a prospective, controlled study. Urology 2002;60:846-50.

15 Lane JA, Hamdy FC, Martin RM, Turner EL, Neal DE, Donovan JL. Latest results from the UK trials evaluating prostate cancer screening and treatment: the CAP and ProtecT studies. Eur J Cancer 2010;46:3095-101.

16 Donovan JL, Mills N, Smith M, Brindle L, Jacoby A, Peters T, et al. Improving design and conduct of randomised trials by embedding them in qualitative research: ProtecT (prostate testing for cancer and treatment) study. BMJ 2002;325:766-70.

17 Donovan JL, Peters TJ, Abrams P, Brookes ST, de la Rosette JJ, Schafer W. Scoring the short form ICSmaleSF questionnaire. J Urol 2000;164:1948-55.

18 Avery K, Donovan J, Peters TJ, Shaw C, Gotoh M, Abrams P. ICIQ: a brief and robust measure for evaluating the symptoms and impact of urinary incontinence. Neurourol Urodyn 2004;23:322-30.

19 Litwin MS, Hays RD, Fink A, Ganz PA, Leake B, Brook RH. The UCLA Prostate Cancer Index: development, reliability, and validity of a health-related quality of life measure. Med Care 1998;36:1002-12.

20 Rosario DJ, Lane JA, Metcalfe C, Catto JW, Dedman D, Donovan JL, et al. Contribution of a single repeat PSA test to prostate cancer risk assessment: experience from the ProtecT study. Eur Urol 2008;53:777-84.

21 Eskicorapci SY, Baydar DE, Akbal C, Sofikerim M, Gunay M, Ekici S, et al. An extended 10-core transrectal ultrasonography guided prostate biopsy protocol improves the detection of prostate cancer. Eur Urol 2004;45:444-8.

22 Trotti A, Bentzen SM. The need for adverse effects reporting standards in oncology clinical trials. J Clin Oncol 2004;22:19-22.

23 Welch HG, Fisher ES, Gottlieb DJ, Barry MJ. Detection of prostate cancer via biopsy in the Medicare-SEER population during the PSA era. J Natl Cancer Inst 2007;99:1395-400.

24 Keetch DW, Catalona WJ, Smith DS. Serial prostatic biopsies in men with persistently elevated serum prostate specific antigen values. J Urol 1994;151:1571-4.

25 Hambrock T, Somford DM, Hoeks C, Bouwense SA, Huisman H, Yakar D, et al. Magnetic resonance imaging guided prostate biopsy in men with repeat negative biopsies and increased prostate specific antigen. J Urol 2010;183:520-7.

26 Braun KP, Brookman-Amissah S, May M, Grassmel Y, Heintze C, Hoschke B, et al. The significance of rebiopsy in the diagnosis of prostate cancer. Urologe A 2009;48:163-9.

27 Herranz AF, Rodriguez FE, Cordero JM, Lledo GE, Verdu TF, Gonzalez CF, et al. [Morbidity of and tolerance to ultrasonography-guided transrectal biopsy of the prostate.] [Spanish.] Acta Urol Esp 1996;20:858-66.

28 Tiong HY, Liew LC, Samuel M, Consigliere D, Esuvaranathan K. A meta-analysis of local anesthesia for transrectal ultrasound-guided biopsy of the prostate. Prostate Cancer Prostatic Dis 2007;10:127-36.

29 Hergan L, Kashefi C, Parsons JK. Local anesthetic reduces pain associated with transrectal ultrasound-guided prostate biopsy: a meta-analysis. Urology 2007;69:520-5. 


\section{What is already known on this topic}

Prostate biopsy is essential for diagnosis of prostate cancer, but its acceptability and effects have rarely been investigated prospectively Prostate biopsy is thought to be well tolerated, but little is known about the effect of adverse events on men, their attitude to re-biopsy, or their subsequent use of healthcare resources

Prostate biopsy can be associated with considerable morbidity, including sepsis, pain, bleeding, and even mortality, but at unknown rates owing to variable reporting

\section{What this study adds}

At seven days after biopsy, 39\% of men had pain, $12 \%$ had fever, $64 \%$ had haematuria, $33 \%$ had rectal bleeding, and $94 \%$ of those who were sexually active had haemoejaculate; all symptoms were less prevalent at 35 days

Men rated only a small proportion of these events as serious, with the exception of haemoejaculate in those who were sexually active, with one in four describing this as a moderate/major problem

Immediately after a first biopsy, one in 10 men had an unfavourable attitude to a repeat procedure, increasing to one in five at seven days

After a first biopsy, one in 10 men sought help from primary care, and one in 100 needed hospital admission within 35 days of the procedure

30 Ingber MS, Ibrahim I, Turzewski C, Hollander JB, Diokno AC. Does periprostatic block reduce pain during transrectal prostate biopsy? A randomized, placebo-controlled, double-blinded study. Int Urol Nephrol 2010;42:23-7.

31 Wolf JS Jr, Bennett CJ, Dmochowski RR, Hollenbeck BK, Pearle MS, Schaeffer AJ, et al. Best practice policy statement on urologic surgery antimicrobial prophylaxis. J Urol 2008:179:1379-90.

32 Aron M, Rajeev TP, Gupta NP. Antibiotic prophylaxis for transrectal needle biopsy of the prostate: a randomized controlled study. BJU Int 2000;85:682-5.

33 Kapoor DA, Klimberg IW, Malek GH, Wegenke JD, Cox CE, Patterson AL, et al. Single-dose oral ciprofloxacin versus placebo for prophylaxis during transrectal prostate biopsy. Urology 1998;52:552-8.

34 Schaeffer AJ, Montorsi F, Scattoni V, Perroncel R, Song J, Haverstock DC, et at. Comparison of a 3-day with a 1-day regimen of an extended-release formulation of ciprofloxacin as antimicrobial prophylaxis for patients undergoing transrectal needle biopsy of the prostate. BJU Int 2007;100:51-7.

35 Nam RK, Saskin R, Lee Y, Liu Y, Law C, Klotz LH, et al. Increasing hospital admission rates for urological complications after transrectal ultrasound guided prostate biopsy. $J$ Urol 2010;183:963-8.

36 Sexton WJ. Editorial comment. J Urol 2010;183:969.

37 Cannon GM Jr, Smaldone MC, Paterson DL. Extended-spectrum beta-lactamase gram-negative sepsis following prostate biopsy: implications for use of fluoroquinolone prophylaxis. Can J Urol 2007:14:3653-5.
38 Carlson WH, Bell DG, Lawen JG, Rendon RA. Multi-drug resistant E coli urosepsis in physicians following transrectal ultrasound guided prostate biopsies-three cases including one death. Can J Urol 2010;17:5135-7.

39 Chappell B, O'Brien T. Infections after prostate biopsy and antibiotic resistance. BJU Int 2009;103:409.

40 Thompson PM, Philpott-Howard J, Wang W. Is the current antibiotic prophylaxis policy in transrectal prostate biopsy still safe? BJU Int 2009;104:271-2.

41 Burden HP, Ranasinghe W, Persad R. Antibiotics for transrectal ultrasonography-guided prostate biopsy: are we practising evidence-based medicine? BJU Int 2008;101:1202-4.

42 Gallina A, Suardi N, Montorsi F, Capitanio U, Jeldres C, Saad F, et al. Mortality at 120 days after prostatic biopsy: a population-based study of 22,175 men. Int $J$ Cancer 2008; 123:647-52.

Accepted: 13 October 2011

\section{Cite this as: BMJ 2012;344:d7894}

This is an open-access article distributed under the terms of the Creative Commons Attribution Non-commercial License, which permits use, distribution, and reproduction in any medium, provided the original work is properly cited, the use is non commercial and is otherwise in compliance with the license. See: http://creativecommons.org/licenses/bync/2.0/ and http://creativecommons.org/licenses/by-nc/2.0/legalcode. 


\section{Tables}

Table 1/ Summary statistics for baseline measures overall and at each centre

\begin{tabular}{|c|c|c|c|c|c|c|c|c|c|c|}
\hline \multirow[b]{2}{*}{ Characteristics } & \multirow{2}{*}{$\begin{array}{l}\text { Total } \\
\text { cohort }\end{array}$} & \multicolumn{8}{|c|}{ Centre number } & \multirow{2}{*}{$\begin{array}{l}\mathrm{P} \text { value for } \\
\text { between } \\
\text { centre } \\
\text { differences }\end{array}$} \\
\hline & & 1 & 2 & 3 & 4 & 5 & 6 & 7 & 8 & \\
\hline No of biopsies & 1147 & 282 & 206 & 176 & 149 & 115 & 102 & 56 & 61 & \\
\hline $\begin{array}{l}\text { Mean (SD) age } \\
\text { (years) }\end{array}$ & $62.1(5.1)$ & $62.0(5.0)$ & $62.8(4.6)$ & $61.2(5.4)$ & $61.8(5.1)$ & $61.9(4.9)$ & $62.5(5.1)$ & $62.2(5.7)$ & $62.9(5.1)$ & $0.09^{*}$ \\
\hline $\begin{array}{l}\text { Median (interquartile } \\
\text { range) PSA (ng/mL) }\end{array}$ & $\begin{array}{c}4.2(3.5 \text { to } \\
5.8)\end{array}$ & $\begin{array}{c}4.3(3.5 \text { to } \\
5.8)\end{array}$ & $\begin{array}{c}4.3(3.5 \text { to } \\
6.1)\end{array}$ & $\begin{array}{c}4.2(3.4 \text { to } \\
5.4)\end{array}$ & $\begin{array}{c}4.2(3.5 \text { to } \\
5.8)\end{array}$ & $\begin{array}{c}4.1 \text { (3.5 to } \\
5.8)\end{array}$ & $\begin{array}{c}4.3(3.5 \text { to } \\
5.6)\end{array}$ & $\begin{array}{c}4.8 \text { (3.6 to } \\
6.9)\end{array}$ & $\begin{array}{c}4.1(3.4 \text { to } \\
5.3)\end{array}$ & $0.69 \dagger$ \\
\hline $\begin{array}{l}\text { Mean }(\mathrm{SD}) \text { body mass } \\
\text { index }\left(\mathrm{kg} / \mathrm{m}^{2}\right)\end{array}$ & $27.4(3.6)$ & $27.3(3.8)$ & $27.1(3.3)$ & $27.9(3.4)$ & $27.1(3.6)$ & $27.8(3.8)$ & $27.7(3.7)$ & $28.1(4.4)$ & $27.5(3.2)$ & $0.54^{*}$ \\
\hline $\begin{array}{l}\text { Mean (SD) HADS } \\
\text { (anxiety) }\end{array}$ & $4.9(3.3)$ & $4.8(3.2)$ & $5.0(3.4)$ & $5.2(3.3)$ & $4.8(3.6)$ & $4.6(2.7)$ & $4.9(3.2)$ & $4.5(2.9)$ & $4.9(3.5)$ & $0.83^{*}$ \\
\hline $\begin{array}{l}\text { Mean (SD) HADS } \\
\text { (depression) }\end{array}$ & $3.1(2.7)$ & $3.2(2.5)$ & $3.3(2.7)$ & $3.4(2.8)$ & $3.0(2.8)$ & $2.7(2.5)$ & $3.1(2.4)$ & $2.4(2.2)$ & $3.3(2.9)$ & $0.2^{*}$ \\
\hline
\end{tabular}

Median (interquartile

range) prostate

volume $(\mathrm{mL})$

HADS=Hospital Anxiety and Depression Scale; PSA=prostate specific antigen.

*From ordinary least squares regression.

†From non-parametric comparison of medians. 


\begin{tabular}{|c|c|c|c|c|}
\hline \multirow[b]{2}{*}{ Symptom reported } & \multicolumn{2}{|c|}{ Symptom present } & \multicolumn{2}{|c|}{ Moderate/serious problem (MS) ${ }^{*}$} \\
\hline & Reporting/respondents & $\%(95 \% \mathrm{Cl})$ & MS/respondents & $\%(95 \% \mathrm{Cl})$ \\
\hline \multicolumn{5}{|l|}{ Within 7 days } \\
\hline Pain & $425 / 1089$ & 39.0 (36.2 to 42.4$)$ & $62 / 1085$ & 5.7 (4.4 to 7.3$)$ \\
\hline Fever & $128 / 1090$ & $11.7(10.0$ to 13.8$)$ & $44 / 1088$ & $4.0(3.0$ to 5.4$)$ \\
\hline Shivers & $135 / 1089$ & $12.4(10.6$ to 14.5$)$ & $35 / 1086$ & $3.2(2.3$ to 4.5$)$ \\
\hline Haematuria & $693 / 1085$ & $63.9(61.0$ to 66.7$)$ & $52 / 1074$ & 4.8 (3.6 to 6.3$)$ \\
\hline Haematochezia & $354 / 1076$ & 32.9 (30.0 to 35.8$)$ & $18 / 1061$ & 1.7 (1.0 to 2.7$)$ \\
\hline Haemoejaculate† & $645 / 747$ & $86.3(83.7$ to 88.6$)$ & $148 / 740$ & $20.0(17.2$ to 23.1$)$ \\
\hline $\begin{array}{l}\text { Any infective/haemorrhagic } \\
\text { symptom } \ddagger\end{array}$ & $691 / 735$ & 94.0 (92.1 to 95.5$)$ & $160 / 714$ & 22.4 (19.5 to 25.6$)$ \\
\hline $\begin{array}{l}\text { Any infective/haemorrhagic } \\
\text { symptom§ }\end{array}$ & $936 / 1047$ & 89.4 (87.4 to 91.1$)$ & $196 / 1013$ & 19.3 (17.0 to 21.9$)$ \\
\hline \multicolumn{5}{|l|}{ Within 35 days $\mathbb{1}$} \\
\hline Pain & $429 / 984$ & 43.6 (40.5 to 46.7$)$ & $71 / 977$ & $7.3(5.7$ to 9.1$)$ \\
\hline Fever & $172 / 985$ & $17.5(15.2$ to 20.0$)$ & $54 / 981$ & $5.5(4.2$ to 7.1$)$ \\
\hline Shivers & $185 / 985$ & 18.8 (16.5 to 21.3 ) & 49/979 & 5.0 (3.7 to 6.6$)$ \\
\hline Haematuria & $642 / 976$ & 65.8 (62.7 to 68.7$)$ & $59 / 958$ & $6.2(4.7$ to 7.9$)$ \\
\hline Haematochezia & $356 / 967$ & 36.8 (33.8 to 39.9 ) & $24 / 951$ & 2.5 (1.6 to 3.7$)$ \\
\hline Haemoejaculate $†$ & $605 / 653$ & 92.6 (90.4 to 94.4$)$ & $172 / 646$ & 26.6 (23.3 to 30.2 ) \\
\hline $\begin{array}{l}\text { Any infective/haemorrhagic } \\
\text { symptom } \ddagger\end{array}$ & $622 / 642$ & 96.9 (95.2 to 98.0$)$ & $181 / 610$ & 29.7 (26.2 to 33.4 ) \\
\hline $\begin{array}{l}\text { Any infective/haemorrhagic } \\
\text { symptom§ }\end{array}$ & $881 / 937$ & 94.0 (92.3 to 95.4$)$ & $240 / 887$ & 27.1 (24.2 to 30.1$)$ \\
\hline
\end{tabular}

*Presence of symptom causing moderate or severe problem.

†Excludes 339 men reporting no sexual activity at either 7 or 35 day assessment.

fOne or more of fever, shivers, haematuria, haematochezia, and haemoejaculate, excluding men reporting no sexual activity at either 7 or 35 day assessment. §One or more of fever, shivers, haematuria, haematochezia, and haemoejaculate, including men reporting no sexual activity at either 7 or 35 day assessment. IIncludes only men with evaluable data for both 7 day and 35 day assessments. 


\begin{tabular}{|c|c|c|c|c|c|c|c|}
\hline \multirow[b]{3}{*}{ Centre } & \multirow[b]{3}{*}{ No of biopsies } & \multicolumn{4}{|c|}{ Immediate report } & & \\
\hline & & \multicolumn{2}{|c|}{ Pain } & \multicolumn{2}{|c|}{ Problem with re-biopsy } & \multicolumn{2}{|c|}{7 day report: problem with re-biopsy } \\
\hline & & MS/response ${ }^{*}$ & $\%(95 \% \mathrm{Cl})$ & MS/response $^{*}$ & $\%(95 \% \mathrm{Cl})$ & MS/response ${ }^{*}$ & $\%(95 \% \mathrm{Cl})$ \\
\hline 1 & 282 & $21 / 282$ & 7 (5 to 11$)$ & $23 / 281$ & $8(6$ to 12 & $30 / 270$ & 11 (8 to 15$)$ \\
\hline 2 & 206 & $37 / 205$ & 18 (13 to 24$)$ & $22 / 206$ & 11 (7 to 16$)$ & $38 / 192$ & 20 (15 to 26$)$ \\
\hline 3 & 176 & $63 / 174$ & 36 (30 to 44$)$ & $37 / 176$ & 21 (16 to 28 ) & $50 / 168$ & 30 (23 to 37 ) \\
\hline 4 & 149 & 9/147 & 6 (3 to 11$)$ & 9/147 & 6 (3 to 11$)$ & $21 / 136$ & 15 (10 to 23 ) \\
\hline 5 & 115 & $16 / 113$ & 14 (9 to 22$)$ & 9/113 & 8 (4 to 14$)$ & $23 / 109$ & 21 (15 to 30 ) \\
\hline 6 & 102 & $11 / 102$ & $11(6$ to 18$)$ & 9/102 & 9 (5 to 16$)$ & $17 / 96$ & $18(11$ to 27$)$ \\
\hline 7 & 56 & $8 / 56$ & 14 (7 to 26$)$ & $8 / 56$ & 14 (7 to 26$)$ & $19 / 55$ & 35 (23 to 48 ) \\
\hline 8 & 61 & $7 / 61$ & 11 (6 to 22 ) & $7 / 61$ & 11 (6 to 22 ) & $15 / 59$ & 25 (16 to 38$)$ \\
\hline Total & 1147 & $172 / 1140$ & $15.1(13.1$ to 17.3$)$ & $124 / 1142$ & 10.9 (9.2 to 12.8$)$ & $213 / 1085$ & 19.6 (17.4 to 22.1$)$ \\
\hline$P$ value $\dagger$ & & & 0.001 & & .001 & & 001 \\
\hline
\end{tabular}

${ }^{*}$ Number of men reporting moderate or severe problem/number responding.

†Age adjusted test of null hypothesis (no between centre difference). 
Table 4| Associations between factors assessed at time of biopsy or at 7 day assessment (cross sectional analysis) and reporting moderate or major problem with re-biopsy $\mathbf{7}$ days after biopsy and contact with health services for biopsy related problem within $\mathbf{3 5}$ days of biopsy

\begin{tabular}{|c|c|c|c|c|c|}
\hline \multirow[b]{2}{*}{ Factors } & \multirow{2}{*}{$\begin{array}{c}\text { Summary statistics in predictors for } \\
\text { overall cohort }\end{array}$} & \multicolumn{2}{|c|}{ Problem with re-biopsy } & \multicolumn{2}{|c|}{ Healthcare contact } \\
\hline & & Odds ratio $(95 \% \mathrm{Cl})$ & P value ${ }^{*}$ & Odds ratio $(95 \% \mathrm{Cl})$ & P value ${ }^{*}$ \\
\hline Pain of biopsy & $172 / 1140(15.1 \%)$ & 8.20 (5.54 to 12.13$)$ & $<0.001$ & 1.81 (1.13 to 2.90$)$ & 0.017 \\
\hline Infective symptoms at 7 days & $47 / 1083(4.3 \%)$ & $7.89(4.16$ to 14.94$)$ & $<0.001$ & 16.78 (8.73 to 32.27$)$ & $<0.001$ \\
\hline Haemorrhagic symptoms at 7 days & 179/1022 (17.5\%) & 4.24 (2.92 to 6.15$)$ & $<0.001$ & 2.89 (1.88 to 4.44$)$ & $<0.001$ \\
\hline Age (odds ratio per 5 years) & Mean 62.1 (SD 5.1) years $(n=1147)$ & $0.86(0.75$ to 1.00$)$ & 0.056 & $1.11(0.93$ to 1.34$)$ & 0.25 \\
\hline Prostate volume (odds ratio per $20 \mathrm{~mL}$ ) & Mean $43.5(\mathrm{SD} 22.8) \mathrm{mL}(\mathrm{n}=1062)$ & $0.82(0.69$ to 0.99$)$ & 0.027 & $1.16(0.99$ to 1.37$)$ & 0.072 \\
\hline History of urinary tract infection & $81 / 1097(7.4 \%)$ & $0.96(0.52$ to 1.79$)$ & 0.91 & 1.99 (1.08 to 3.65$)$ & 0.036 \\
\hline History of prostatitis & $18 / 1094(1.6 \%)$ & 1.41 (0.47 to 4.18$)$ & 0.55 & 2.99 (1.02 to 8.76$)$ & 0.065 \\
\hline Positive midstream urine at biopsy & $18 / 1078(1.7 \%)$ & $1.15(0.32$ to 4.15$)$ & 0.84 & 2.69 (0.93 to 7.80$)$ & 0.091 \\
\hline Warfarin treatment & $14 / 1091(1.3 \%)$ & $0.38(0.05$ to 3.00$)$ & 0.30 & 2.99 (0.91 to 9.85$)$ & 0.097 \\
\hline Haematuria/clots in first voided urine & $81 / 1055(7.7 \%)$ & 1.04 (0.59 to 1.85$)$ & 0.89 & 1.12 (0.56 to 2.22$)$ & 0.75 \\
\hline Aspirin treatment & $244 / 1096(22.3 \%)$ & 0.87 (0.58 to 1.29$)$ & 0.48 & 0.87 (0.54 to 1.39$)$ & 0.55 \\
\hline NSAID treatment & $58 / 1093(5.3 \%)$ & $0.68(0.32$ to 1.43$)$ & 0.29 & $0.85(0.35$ to 2.04$)$ & 0.71 \\
\hline
\end{tabular}

Odds ratios and $P$ values are adjusted for age and centre.

NSAID=non-steroidal anti-inflammatory drug.

${ }^{*}$ Calculated with likelihood ratio tests. 